\title{
Molecular Species of Diacylglycerols and Phosphoglycerides and the Postmortem Changes in the Molecular Species of Diacylglycerols in Rat Brains
}

\author{
Chunghee Lee and Amiya K. Hajra \\ Neuroscience Laboratory, Mental Health Research Institute; and Department of Biological Chemistry, \\ University of Michigan, Ann Arbor, Michigan, U.S.A.
}

\begin{abstract}
The molecular species of 1,2-diacyl-sn-glycerol (DAG), phosphatidylcholine (PC), phosphatidylethanolamine (PE), phosphatidylserine (PS), phosphatidylinositol (PI), phosphatidylinositol 4-phosphate (PIP), and phosphatidylinositol 4,5-bisphosphate $\left(\mathrm{PIP}_{2}\right)$ from brains of adult rats (weighing $150 \mathrm{~g}$ ) were determined. The DAG, isolated from brain lipid extracts by TLC, was benzoylated, and the molecular species of the purified benzoylated derivatives were separated from each other by reverse-phase HPLC. The total amount and the concentration of each species were quantified by using 1,2-distearoyl-sn-glycerol (18:0-18:0) as an internal standard. About 30 different molecular species containing different fatty acids at the $s n-1$ and $s n-2$ positions of DAG were identified in rat brains ( $1 \mathrm{~min}$ postmortem), and the predominant ones were 18:0-20:4 (35\%), 16:0-18:1 (15\%), 16:0-16:0 (9\%), and 16:0-20:4 (8\%). The molecular species of PC, PE, PS, and PI were determined by hydrolyzing the lipids with phospholipase $C$ to DAG, which was then benzoylated and subjected to reverse-phase HPLC. PIP and PIP were first dephosphorylated to PI with alkaline phosphatase before hydrolysis by phospholipase $C$. The molecular species composition of phosphoinositides showed predominantly the 18:0-20:4 species $\left(50 \%\right.$ in PI and $\sim 65 \%$ in PIP and PIP $_{2}$ ).
\end{abstract}

PS contained mainly the 18:0-22:6 (42\%) and 18:0-18:1 (24\%) species. PE was mainly composed of the $18: 0-20: 4(22 \%), 18$ : 0-22:6 (18\%), 16:0-18:1 (15\%), and 18:0-18:1 (15\%) species. In PC the main molecular species were 16:0-18:1 (36\%), 16: 0-16:0 (19\%), and 18:0-18:1 (14\%). Studies on postmortem brains ( $30 \mathrm{~s}$ to $30 \mathrm{~min}$ ) showed a rapid increase in the total amount (from $40-50 \mathrm{nmol} / \mathrm{g}$ in $0 \mathrm{~min}$ to $210-290 \mathrm{nmol} / \mathrm{g}$ in $30 \mathrm{~min}$ ) and in all the molecular species of DAG. Comparatively larger increases (seven- to 10 -fold) were found for the 18:0-20:4 and 16:0-20:4 species. Comparison of DAG species with the molecular species of different glycerolipids indicated that the rapid postmortem increase in content of DAG was mainly due to the breakdown of phosphoinositides. However, a slow but continuous breakdown of PC to DAG was also observed. Key Words: 1,2-Diacyl-snglycerol - Phosphatidylcholine - Phosphatidylethanolamine-Phosphatidylserine - Phosphatidylinositol-Phosphatidylinositol phosphate-Phosphatidylinositol bisphosphate-Rat-Brain. Lee C. and Hajra A. K. Molecular species of diacylglycerols and phosphoglycerides and the postmortem changes in the molecular species of diacylglycerols in rat brains. J. Neurochem. 56, 370-379 (1991).
Diacylglycerol (1,2-diacyl-sn-glycerol; DAG) has long been known as an important intermediate for glycerolipid biosynthesis in eukaryotic cells (Kennedy, 1957). This biosynthetic precursor is formed in endoplasmic reticulum by hydrolysis of phosphatidic acid (PA), and then it is converted to triglycerides or phosphoglycerides in the same subcellular compartment (Bell and Coleman, 1980). In recent years DAG has also been shown to be an important effector for a transmembrane signaling system elicited by different hormones and neurotransmitters (Nishizuka, 1984, 1986). It is postulated that plasma membrane phosphoinositides are hydrolyzed to DAG by a receptormediated activation of phospholipase $\mathrm{C}$ and that this DAG, in turn, activates protein kinase C. DAG, generated from inositides, has also been postulated to be the source of free arachidonic acid (20:4), which is the precursor of all eicosanoids (Prescott and Majerus, 1983). These biomessenger roles of DAG were amply verified in several in vitro systems where phosphoino-
Received March 30, 1990; revised manuscript received June 18, 1990; accepted June 26, 1990.

Address correspondence and reprint requests to Dr. A. K. Hajra at Neuroscience Laboratory, University of Michigan, 1103 East Huron Street, Ann Arbor, MI 48104-1687, U.S.A.
Abbreviations used: DAG, 1,2-diacyl-sn-glycerol; PA, phosphatidic acid; PC, phosphatidylcholine; PE, phosphatidylethanolamine; PI, phosphatidylinositol; PIP, phosphatidylinositol 4-phosphate; $\mathrm{PIP}_{2}$, phosphatidylinositol 4,5-bisphosphate; 4-PP, 4-pyrrolidinopyridine; PS, phosphatidylserine. 
sitides were prelabeled by using different radioactive precursors and the radioactive products were analyzed (Nishizuka, 1986). An increase in the mass of DAG in different systems after stimulation by different ligands has also been demonstrated (Loeffelholz, 1989; Pelech and Vance, 1988). However, the source(s) and subcellular site(s) of such receptor-mediated generation of DAG have not been firmly established. Recently phosphatidylcholine (PC) has been postulated to be the source of DAG in several signal-transducing systems (Exton, 1990).

One way to distinguish such receptor-mediated formation and metabolism of DAG from de novo biosynthetic DAG is to analyze the molecular species of DAG in these systems. The phosphoinositides in most systems have been shown to be comprised mostly (60$80 \%$ ) of one single molecular species, i.e., 1-stearoyl2-arachidonoyl-sn-glycerol derivative, and therefore the DAG formed from inositides should consist of the same species. In contrast, the biosynthetic DAG or the DAG from sources other than inositides should contain different fatty acids. Therefore, an analysis of the molecular species of DAG may provide some indications regarding its source and possible site of formation. This has been attempted by some workers who used a sensitive radiometric method in which the DAG was converted to ${ }^{32} \mathrm{P}$-phosphatidate by [ $\left.{ }^{32} \mathrm{P}\right] \mathrm{ATP}$ in the presence of either endogenous or exogenous DAG kinase and then the ${ }^{32} \mathrm{P}$-labeled phosphatidates (as dimethyl esters) were separated from each other by either argentation TLC or HPLC (Van Rooijen et al., 1985; Kennerly, 1987). However, a good separation of different molecular species of phosphatidate was not achieved by these methods.

The present work was undertaken to develop a sensitive method for the quantitative analysis of molecular species of DAG in different biological systems. The methodology was based on the demonstration by several workers that reverse-phase HPLC could be used for good resolution of different molecular species of DAG (generated from hydrolysis of phosphoglycerides by phospholipase C) after acylation to the acetyl (Nakagawa and Horrocks, 1983), benzoyl (Blank et al., 1984), or other derivatives (Batley et al., 1980; Krueger et al., 1984). We have adapted these methods, especially that described for the reverse-phase HPLC separation of benzoyl DAG, and used an internal standard to quantify different molecular species of DAG present in rat brain.

DAG has been shown to be present in small quantities in brain, and it has been shown that there is a rapid postmortem increase in its amount in brain (Banschbach and Geison, 1974; Aveldano and Bazan, 1975; Ikeda et al., 1986). A preponderance of 18:0 and 20:4 fatty acids in this DAG indicated that the rapid postmortem increase in its level was probably due to the hydrolysis of brain phosphoinositides. We have verified this directly by quantifying the postmortem changes in the molecular species of rat brain DAG and also have analyzed the molecular species of different phosphoglycerides, including the inositides. These results are presented here.

\section{MATERIALS AND METHODS}

\section{Materials}

Sprague-Dawley rats (5 weeks old, weighing 150-160 g) were obtained from Harlan Laboratories (Indianapolis, IN, U.S.A.). Benzoic anhydride, 4-pyrrolidinopyridine (4-PP), 1,2-distearoyl-sn-glycerol, phospholipase C (Bacillus cereus), and calf intestine alkaline phosphatase were obtained from Sigma Chemical Co. (St. Louis, MO, U.S.A.). PUFA-2, a GLC standard mixture of polyunsaturated fatty acids, was obtained from Supelco (Bellefonte, PA, U.S.A.). All the solvents for HPLC were HPLC grade, and other solvents were chemical grade. TLC plates (silica gel 60) were purchased from Merck (Cherry Hill, NJ, U.S.A.). Dry benzene was prepared by distilling it from calcium hydride. 1-Stearoyl-2$\left[5,6,8,9,11,12,14,15-{ }^{3} \mathrm{H}\right]$ arachidonoyl-sn-glycerophosphoryl choline was purchased from NEN-Dupont (Boston, MA).

\section{Extraction and purification of DAG}

Rats were anesthetized with diethyl ether, and their forebrains (cerebrum and cerebellum) were quickly excised and homogenized in chloroform/methanol (1:1, $12 \mathrm{ml} / \mathrm{g}$ of tissue) with a Polytron (Brinkmann) homogenizer. A known amount $(\sim 30 \mathrm{nmol} / \mathrm{g}$ of brain) of internal standard, i.e., 1,2-distearoyl-sn-glycerol, was added to the homogenate. The homogenate was centrifuged at low speed $(1,000 \mathrm{~g}, 10 \mathrm{~min})$, and the supernatant was saved. The residue was dispersed in water $(0.5 \mathrm{ml} / \mathrm{g}$ of tissue) by agitation in a sonic bath, and this dispersion was rehomogenized in chloroform/methanol (1: $1,6 \mathrm{ml} / \mathrm{g}$ of original tissue). This second homogenate was centrifuged $(1,000 \mathrm{~g}, 10 \mathrm{~min})$, the supernatant was combined with the first supernatant, and the residue was saved for acidic extraction (see below). To the combined supernatants, an additional amount of chloroform was added ( $9 \mathrm{ml} / \mathrm{g}$ of tissue) and mixed well, and $0.9 \%$ aqueous $\mathrm{NaCl}(5.5 \mathrm{ml} / \mathrm{g}$ of tissue) was added to the extract to separate the phases (Folch et al., 1957). The mixture was vortex-mixed and centrifuged $(1,000$ $g, 5 \mathrm{~min}$ ), and the upper layer was removed. The lower layer was dried in a rotary evaporator, and the residue was dissolved in chloroform (3.5 ml/g of tissue) and, when necessary, stored at $-20^{\circ} \mathrm{C}$ under nitrogen. This lipid extract by a neutral solvent mixture was spotted on a $20-\times 20-\mathrm{cm}$ TLC plate (Merck silica gel $60,0.25 \mathrm{~mm}$ thick) as a band (lipid extract from $\sim 10 \mathrm{mg}$ of tissue $/ \mathrm{cm}$ ), and the plate was developed with toluene/ether/methanol (80:10:10 by volume) up to $10 \mathrm{~cm}$ from the origin. The DAG band $\left(R_{\mathrm{f}}=0.60\right)$ was identified by comparison with the standard DAG chromatographed side-by-side after spraying with Primuline $(0.001 \%)$ (Wright, 1971 ) and viewing under UV light. The band was scraped off, and the DAG was extracted three times from TLC powder with $2 \mathrm{ml}$ of diethyl ether by sonication for $30 \mathrm{~s}$ followed by centrifugation $(1,000 \mathrm{~g}, 5 \mathrm{~min})$. The ether was removed by blowing $\mathrm{N}_{2}$, and the DAG was used immediately for benzoylation.

\section{Purification of other lipids}

Phospholipids [PC, phosphatidylethanolamine (PE), phosphatidylserine (PS), and phosphatidylinositol (PI)] present in the neutral extract were separated from each other by TLC using two developments in the solvent system of chloroform $/$ methanol $/ 0.25 \% \mathrm{KCl} /$ ethyl acetate $/ n$-propanol (30: 
9:6:18:25 by volume) (Hedegaard and Jensen, 1981). The bands were visualized by spraying with Primuline and identified by comparison with authentic standards (chromatographed side-by-side). The bands were scraped off, and the lipids were extracted from the powders with either chloroform $/$ methanol $(2: 1 \mathrm{vol} / \mathrm{vol})$ for PC, PS, and PE or chloroform $/$ methanol $(2: 1 \mathrm{vol} / \mathrm{vol})$ containing $0.25 \% \mathrm{HCl}$ for $\mathrm{PI}$. The $\mathrm{HCl}$ was removed by adding 0.25 volume of water to the extract to separate the phases, the upper layer was removed, and the lower layer was dried by blowing $\mathrm{N}_{2}$.

Polyphosphoinositides were isolated from the tissue residues, left after neutral chloroform/methanol extraction, by extracting with chloroform/methanol/12 $M \mathrm{HCl}$ (200:100: 0.75 by volume, $16 \mathrm{ml} / \mathrm{g}$ of brain) and centrifuged, and the supernatant was transferred to another tube. To the supernatant 0.25 volume of $1 \mathrm{M} \mathrm{HCl}$ was added, mixed well, and centrifuged. The upper layer was removed, and the lower phase was washed once with Folch upper phase (Folch et al., 1957) and then evaporated to dryness by blowing $\mathrm{N}_{2}$. The residue was dissolved in $2 \mathrm{ml}$ of chloroform/methanol/water (75:25:2 by volume), and the extract was spotted as a band on a TLC plate impregnated with $1.2 \%$ potassium oxalate (Hajra et al., 1988). The plate was developed with chloroform/ methanol/7.4 $M \mathrm{NH}_{4} \mathrm{OH} /$ water (40:48:5:10 by volume) followed by $n$-propanol/7.4 $M \mathrm{NH}_{4} \mathrm{OH} /$ water $(65: 20: 15$ by volume) (Grove et al., 1981). Bands were localized after spraying with Primuline, and the bands containing PI 4-phosphate (PIP) and PI 4,5-bisphosphate (PIP ${ }_{2}$ ) were extracted from the TLC scrapings by dispersing them three times with $10 \mathrm{ml}$ of chloroform/methanol/12 $\mathrm{MHCl}$ (66.6:33.3:0.25 by volume). To the combined extracts 0.25 volume of water was added, mixed well, and centrifuged, and the upper layer was removed. The lower layer was filtered through a PTFE filter (pore size, $0.45 \mu \mathrm{m}$ ) to remove insoluble material, and the solvents were removed under $\mathrm{N}_{2}$. The dried PIP and $\mathrm{PIP}_{2}$ residues were subjected to enzymatic dephosphorylation to convert them to PI as described below.

\section{Hydrolysis with alkaline phosphatase}

PIP and PIP $_{2}$ are not hydrolyzed by bacterial phospholipase $\mathrm{C}$, and, therefore, these lipids were first dephosphorylated to PI, which could then be converted to DAG. PIP and PIP were hydrolyzed to PI by alkaline phosphatase by the method of Ehle et al. (1985). The lipids were dried down under $\mathrm{N}_{2}$ in Reacti-Vials (Pierce Chemical Co.) and then magnetically stirred in a reaction mixture (final volume, $0.5 \mathrm{ml}$ ) containing $10 \mathrm{mM}$ Tris- $\mathrm{HCl}$ buffer ( $\mathrm{pH} 7.4$ ), sodium deoxycholate (3 $\mathrm{m} M$ ), and alkaline phosphatase $(2 \mathrm{U})$ for $6 \mathrm{~h}$ at $37^{\circ} \mathrm{C}$. The reaction was stopped by adding $5.0 \mathrm{ml}$ of chloroform $/ \mathrm{meth}$ anol $(2: 1 \mathrm{vol} / \mathrm{vol})$ followed by $1 \mathrm{ml}$ of $1 \mathrm{M} \mathrm{HCl}$. After mixing and centrifuging, the lower layer was transferred to another tube and dried under $\mathrm{N}_{2}$, and the PI formed was purified by TLC as described above.

\section{Phospholipase $\mathrm{C}$ hydrolysis}

PC, PI, PS, and PE were sonicated with $0.30 \mathrm{ml}$ of $0.25 \%$

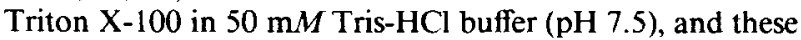
mixed micelles were then incubated with $10 \mathrm{U}$ of phospholipase $\mathrm{C}$ (from B. cereus) at $37^{\circ} \mathrm{C}$ for $6 \mathrm{~h}$ (Sundler et al, 1978). The reaction was terminated by chloroform/methanol/ $1 \mathrm{M} \mathrm{HCl}$ as described above, and the DAG from the lower layer was purified by TLC (toluene/ether/methanol 80:10:10 by volume). Authentic standard 1-stearoyl-2-[ $\left.{ }^{3} \mathrm{H}\right]$ arachidonoyl-sn-glycerol-3-phosphocholine was converted to the corresponding $\left[{ }^{3} \mathrm{H}\right] \mathrm{DAG}$ by using the same procedure.

\section{Benzoylation of DAG}

The purified DAG in dry ether was quantitatively transferred to a Reacti-Vial with dry ether, and the solvent was removed by blowing $\mathrm{N}_{2}$. To the dry DAG $(\sim 10-40 \mathrm{nmol})$ $100 \mu \mathrm{l}$ of dry benzene and $20 \mu \mathrm{l}$ of a solution containing 6.8 $\mu \mathrm{mol}$ of 4-PP and $5.5 \mu \mathrm{mol}$ of benzoic anhydride in dry benzene were added, and the mixture was magnetically stirred under $\mathrm{N}_{2}$ at room temperature for $1 \mathrm{~h}$. The reaction was stopped by adding $0.1 \mathrm{ml}$ of methanol, and the mixture was stirred for another $5 \mathrm{~min}$. The mixture was then spotted on TLC plates as a band and developed with hexane/diethyl ether/7.4 $M \mathrm{NH}_{4} \mathrm{OH}$ (55:45:1 by volume). The benzoyl DAG band $\left(R_{\mathrm{f}}=0.65\right)$ was scraped off, extracted three times with $2 \mathrm{ml}$ of ether, and used for purification by HPLC.

Purification of benzoyl DAG by normal-phase HPLC

The normal-phase HPLC was performed using a Waters M-45 stainless steel solvent delivery system and a silica gel column ( $25 \times 0.46 \mathrm{~cm}$; pore size, $5 \mu \mathrm{m}$; Rainin) attached to a Spectroflow Kratos 773 UV monitoring system. The benzoyl DAG sample in ether was filtered through a PTFE filter (pore size, $0.45 \mu \mathrm{m}$ ) to remove particulate matter, and the solvent was removed by blowing $\mathrm{N}_{2}$. The residue was dissolved in cyclohexane, and a 20- $\mu \mathrm{l}$ aliquot was injected into the column. Benzoyl DAG was eluted isocratically using cyclohexane/ether $(96.3: 3.7 \mathrm{vol} / \mathrm{vol})$ at a flow rate of $1 \mathrm{ml} / \mathrm{min}$ at room temperature. The elution was monitored at $228 \mathrm{~nm}$, and the benzoyl DAG peak was collected and dried un$\operatorname{der} \mathrm{N}_{2}$.

\section{Separation of molecular species of DAG}

The DAG fraction collected from normal-phase HPLC was dried, dissolved in acetonitrile, and injected into an octadecyl silica gel reverse-phase column $(25 \times 0.46 \mathrm{~cm}$; Rainin). The molecular species were eluted isocratically using acetonitrile/isopropanol (70:30 vol/vol) at a flow rate of 1 $\mathrm{ml} / \mathrm{min}$ at room temperature. The eluate was monitored at $228 \mathrm{~nm}$, and the peak areas were integrated using a Nelson analytical PC integrator with a 900 series interface attached to the monitoring system.

Identification of reverse-phase HPLC peaks by GLC

For the purpose of identification, each peak collected from the HPLC column was dried and methanolyzed by heating for $15 \mathrm{~min}$ at $60^{\circ} \mathrm{C}$ with $0.5 \mathrm{ml}$ of $13 \% \mathrm{BF}_{3}$ in methanol. Two milliliters of hexane and $1.5 \mathrm{ml}$ of water were added to the mixture, mixed well, and centrifuged. The upper hexane layer was transferred to another tube, and the lower layer was extracted two more times with $2 \mathrm{ml}$ of hexane. The combined hexane layers after washing with water were dried under $\mathrm{N}_{2}$, dissolved in a small volume of dichloromethane, and injected into the gas chromatographic column.

The gas chromatography was performed using a HewlettPackard gas chromatograph (model 5800) equipped with a fused silica capillary column $(30 \mathrm{~m} \times 0.2 \mathrm{~mm}$ ) coated with SP 2330 (cyanopropyl silicone from Supelco) and a flame ionization detector. The carrier gas was helium, and the area under each peak was determined using a Hewlett-Packard integrator (model 3396A). The peaks were identified by comparing the retention values with those of known standards.

\section{Postmortem changes in the molecular species of DAG}

To analyze the changes of molecular species and amounts of DAG, the rats were lightly anesthetized with ether. For the zero-time points, the skull was opened, and the brain was 
excised with a spatula and immediately $(<10 \mathrm{~s})$ dropped into liquid $\mathrm{N}_{2}$. For the time course study, the rats were decapitated, and the whole heads were opened, put into plastic bags, and incubated at $37^{\circ} \mathrm{C}(>1 \mathrm{~min}$ time course) for the indicated interval $(0.5,1.0,3.0,12$, and $30 \mathrm{~min})$. After incubation, the brains were rapidly excised from the heads and dropped immediately into liquid nitrogen. The frozen brains were homogenized in chloroform/methanol, and the DAG was isolated from the extracts as described above.

\section{RESULTS}

\section{Purification of DAG}

DAG $\left(R_{\mathrm{f}}=0.6\right)$ migrated just above the wide cholesterol band $\left(R_{\mathrm{f}}=0.5\right)$ and was well separated from other neutral lipids or free fatty acid $\left(R_{\mathrm{f}}=0.36\right)$ in the solvent system used for TLC. Recovery of DAG from brain extract was $\sim 80-85 \%$ (Table 1). Alkyl and alkenyl acyl glycerol, if present, were located just above the DAG band.

\section{Conversion of different phospholipids to DAG}

PC, PS, PI, and PE had $R_{\mathrm{f}}$ values of $0.15,0.22,0.28$, and 0.50 , respectively, in the TLC system used. PIP and PIP ${ }_{2}$ had $R_{\mathrm{f}}$ values of 0.21 and 0.33 , respectively, in the TLC system used for the oxalated plates. By TLC examination, it was found that the conversion of PIP and PIP $\mathrm{P}_{2}$ to PI by alkaline phosphatase was complete. By TLC analysis, it was also found that phospholipase $\mathrm{C}$ from $B$. cereus completely hydrolyzed all phospholipids. The phospholipase $\mathrm{C}$ from Clostridium perfringens did not hydrolyze PI but hydrolyzed other lipids.

\section{Benzoylation of DAG and purification of benzoyl DAG}

The benzoylation reaction using 4-PP as the catalyst went to completion within $1 \mathrm{~h}$ (Fig. 1). After the reaction, the excess benzoic anhydride was converted to methyl benzoate by addition of methanol, and the benzoyl DAG was purified by TLC. In the TLC system used, the benzoyl DAG had an $R_{\mathrm{f}}$ value of 0.65 , well separated from both methyl benzoate $\left(R_{\mathrm{f}}=0.9\right)$ and the catalyst $\left(R_{\mathrm{f}}=0\right)$. The recovery of the benzoyl DAG after benzoylation and TLC was 90\% (Table 1).

Benzoyl DAG was further purified by normal-phase HPLC to remove impurities extracted from TLC plates and also to remove small amounts of ether analogs of DAG if they were present. In the HPLC system used, the benzoyl DAG was eluted out as a single peak with a retention time of $13 \mathrm{~min}$. In this system the benzoyl derivative of alkyl acyl glycerol had an $R_{\mathrm{f}}$ value of 6.7 $\mathrm{min}$, and that for alkenyl acyl glycerol was $5.6 \mathrm{~min}$. The presence of ether analogs was noted when DAG was derived from phosphoglycerides, especially from PE. Very little, if any, of the ether analogs of DAG was present in the TLC and HPLC systems used for the isolation of free DAG.

\section{Separation and identification of molecular species of DAG}

The elution profile of purified brain benzoyl DAG from the reverse-phase HPLC column is shown in Fig. 2. There are a total of 18 peaks plus the peak for the internal standard, i.e., benzoylated distearoyl glycerol. This chromatogram was typical of the brain DAG profile obtained 1-2 min after decapitation (see below). From the total area under all the peaks and from the amount of the 1,2-distearoyl-sn-glycerol added as the internal standard and the area of that peak (Fig. 2), it was calculated that the amount of DAG present was $98 \mathrm{nmol} / \mathrm{g}$ of brain for that particular experiment (Table 2).

The peaks were identified by collecting each one from the eluate and then analyzing the fatty acids released after methanolysis by gas chromatography. Peaks containing a single molecular species, e.g., peak 10 , were easily identified by the 1:1 molar proportion of two fatty acids seen after gas chromatography. Peaks containing multiple molecular species, e.g., peak 8 , were identified by the molar proportions of different fatty acids seen after gas chromatography and also by plotting the relative retention time with the effective

TABLE 1. Recovery of labeled DAG at each step of analysis

\begin{tabular}{llccc}
\hline & \multicolumn{2}{c}{ Experiment A } & \multicolumn{2}{c}{ Experiment B } \\
\cline { 2 - 5 } \multicolumn{1}{c}{ Sample } & $\begin{array}{c}\text { Radioactivity } \\
(\mathrm{cpm})\end{array}$ & $\begin{array}{c}\text { Recovery } \\
(\%)\end{array}$ & $\begin{array}{c}\text { Radioactivity } \\
(\mathrm{cpm})\end{array}$ & $\begin{array}{c}\text { Recovery } \\
(\%)\end{array}$ \\
\hline Brain extract & $3.34 \times 10^{5}$ & 100 & $3.34 \times 10^{5}$ & 100 \\
After first TLC & $2.71 \times 10^{5}$ & 81.3 & $2.81 \times 10^{5}$ & 84.2 \\
After second TLC & $2.51 \times 10^{5}$ & 92.4 & $2.54 \times 10^{5}$ & 90.3 \\
DAG peak from normal-phase HPLC & $2.39 \times 10^{5}$ & 95.3 & $2.47 \times 10^{5}$ & 97.1 \\
18:0-20:4 peak from reverse-phase HPLC & $2.37 \times 10^{5}$ & 99.2 & $2.48 \times 10^{5}$ & 100 \\
Overall yield & & 70.9 & & 73.8 \\
\hline
\end{tabular}

To the total brain lipid extract a trace amount of 1-stearoyl-2-[5,6,8,9,1 1,12,14,15- $\left.{ }^{3} \mathrm{H}\right]$ archidonoyl-sn-glycerol was added, and the extract was analyzed for different molecular species of DAG as described in the text. Radioactivity in the aliquots at each stage of purification and in the final HPLC eluate (peak 10 in Fig. 2) was determined. These counts, after correction for the counts taken out in the aliquots, are presented here. 


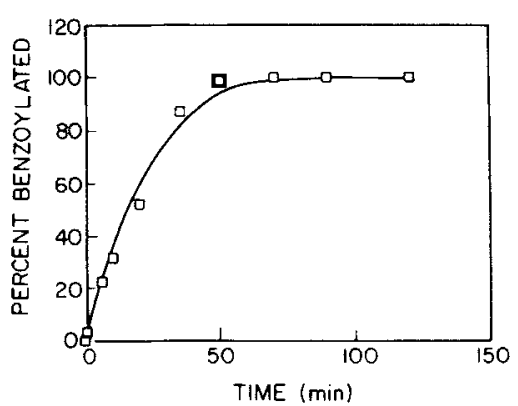

FIG. 1. Time course of benzoylation of DAG with benzoic anhydride. Total DAG $(\sim 100 \mathrm{nmol})$ from one rat brain containing a tracer amount of 1-stearoyl-2-[5,6,8,9,11,12,14,15- $\left.{ }^{3} \mathrm{H}\right]$ arachidonoyl-sn-glycerol $\left(2.76 \times 10^{5} \mathrm{cpm}\right)$ was benzoylated with benzoic anhydride using 4-PP as the catalyst in dry benzene (total volume, $450 \mu \mathrm{l})$ as described in the text. At the indicated times, $30 \mu \mathrm{l}$ of the reaction mixture was taken out and spotted on a TLC plate, which was developed in hexane/ether $/ \mathrm{NH}_{4} \mathrm{OH}$ (55:45:1 by volume). The spots corresponding to DAG $\left(R_{\mathrm{f}}=0.60\right)$ and benzoyl DAG $\left(\boldsymbol{R}_{\mathrm{f}}\right.$ $=0.65$ ) were scraped out, and the radioactivity in the scrapings was determined. The result was given as the percentage of radioactivity present in the benzoyl DAG fraction. Duplicate experiment showed less than approximately $\pm 5 \%$ variability at the initial intervals $(<20 \mathrm{~min})$.

carbon number by the method of Patton et al. (1982). The position assigned for the fatty acids at the $s n-1$ and $s n-2$ position of the glycerol moiety is arbitrary, with the assumption that the more saturated one is present at C-1.

The identities of each peak along with the amount present for a typical chromatogram are given in Table 2. The major peaks seen were 16:0-20:4 (peak 5), 18: 0-20:4 (peak 10), 16:0-16:0 (peak 13), and 16:0-18:1 (peak 12; this peak was mixed with other minute components) (Fig. 2 and Table 2).

\section{Molecular species of brain phosphoglycerides}

The HPLC elution profile of the DAG (benzoylated) obtained from total brain PC is shown in Fig. 3. Elution profiles obtained for PI and $\mathrm{PIP}_{2}$, which were quite different from that for PC, are shown in Figs. 4 and 5, respectively. The peaks are numbered the same way as in Fig. 2, and their identities are the same as in Table 2 . The relative amount (in mol\%) of each peak is given in Table 3. Table 3 also shows the percent composition of molecular species for PE and PS.

\section{Postmortem changes in the molecular species of DAG}

The postmortem increase in the total amount of brain DAG, calculated from the amount of internal standard recovered after HPLC separation, is shown in Fig. 6. Although the amounts of all DAG species increased, this increase was more pronounced for some species than others. The changes in the major molecular species are shown in Fig. 7. The postmortem changes of both relative and absolute amount of each peak as analyzed by HPLC are shown in Table 4 .

\section{DISCUSSION}

Derivatives of DAGs, obtained after phospholipase $C$ hydrolysis of different phospholipids, have been used by different researchers to analyze the molecular species of phosphoglycerides (Batley et al., 1980; Nakagawa and Horrocks, 1983; Blank et al., 1984; Krueger et al., 1984). We found that the acetyl and benzoyl derivatives of DAG were well-suited for the separation of all the molecular species of brain DAG by reverse-phase HPLC. The benzoyl derivatives, as reported by Blank et al. (1984), were chosen for such separation because of the high absorbance of the benzoyl group $\left(1.3 \times 10^{4}\right.$ $M^{-1} \mathrm{~cm}^{-1}$ ), which was the same for all the species regardless of their fatty acid compositions. Several modifications of the published method were made, which included the use of relatively nontoxic solvents for TLC, a different catalyst (4-PP) for benzoylation, and an internal standard (1,2-distearoyl-sn-glycerol) for quantification. A different catalyst was used, because

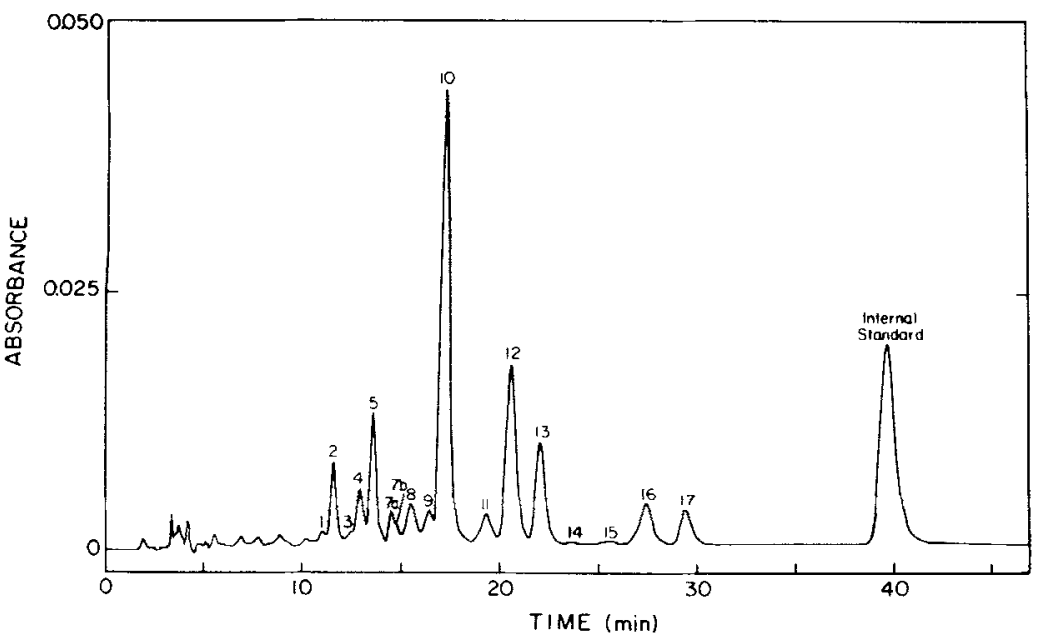

FIG. 2. Separation profile of benzoyl DAG by reverse-phase HPLC. DAG isolated from rat brains ( 1 min postmortem) containing the added internal standard, 1,2-distearoyl-sn-glycerol (30 $\mathrm{nmol} / \mathrm{g}$ brain), was benzoylated, purified, and subjected to reverse-phase HPLC as described in the text. A total of $\sim 20 \mathrm{nmol}$ of benzoyl DAG was injected into the HPLC column. The detector output $\left(A_{228}\right)$ versus time is plotted here. The composition of each numbered peak is given in Table 2 . 
TABLE 2. Molecular species composition of rat brain $D A G$

\begin{tabular}{|c|c|c|c|c|}
\hline Peak & $\begin{array}{l}\text { Retention } \\
\text { time } \\
\text { (min) }\end{array}$ & $\begin{array}{c}\text { Concentration } \\
\text { (nmol/g of brain) }\end{array}$ & $\operatorname{mol} \%$ & DAG species \\
\hline 1 & 10.95 & 0.54 & 0.55 & $18: 1-22: 6(n-3)$ \\
\hline 2 & 11.42 & 4.43 & 4.52 & $16: 0-22: 6(n-3)$ \\
\hline 3 & 12.26 & 0.26 & 0.26 & $18: 1-22: 5(n-3)$ \\
\hline \multirow[t]{2}{*}{4} & 12.77 & 3.69 & 3.76 & $18: 1-20: 4(n-6)$ \\
\hline & & 0.23 & 0.23 & $16: 0-22: 5(n-3)$ \\
\hline 5 & 13.40 & 7.92 & 8.09 & $16: 0-20: 4(n-6)$ \\
\hline 6 & 13.90 & $\mathrm{Tr}$ & $\operatorname{Tr}$ & $18: 0-20: 5(n-3)$ \\
\hline $7 \mathrm{a}$ & 14.37 & 1.51 & 1.54 & $18: 0-22: 6(n-3)$ \\
\hline \multirow[t]{4}{*}{$7 b$} & 14.61 & 0.10 & 0.11 & $16: 1-18: 1$ \\
\hline & & 0.06 & 0.06 & $18: 1-18: 2(n-6)$ \\
\hline & & 0.34 & 0.35 & $18: 1-22: 4(n-6)$ \\
\hline & & 0.09 & 0.09 & $18: 1-20: 3(n-6)$ \\
\hline \multirow[t]{5}{*}{8} & 15.35 & 0.22 & 0.22 & $14: 0-16: 0$ \\
\hline & & 0.88 & 0.90 & $16: 0-16: 1$ \\
\hline & & 0.96 & 0.98 & $16: 0-18: 2(n-6)$ \\
\hline & & 1.01 & 1.03 & $16: 0-22: 4(n-6)$ \\
\hline & & 0.47 & 0.48 & $16: 0-20: 3(n-6)$ \\
\hline 9 & 16.30 & 2.34 & 2.38 & $18: 0-22: 5(n-3)$ \\
\hline 10 & 17.00 & 35.30 & 36.03 & $18: 0-20: 4(n-6)$ \\
\hline 11 & 19.18 & 2.61 & 2.67 & $18: 1-18: 1$ \\
\hline \multirow[t]{4}{*}{12} & 20.38 & 15.00 & 15.31 & $16: 0-18: 1$ \\
\hline & & 0.58 & 0.59 & $18: 0-18: 2(n-6)$ \\
\hline & & 0.84 & 0.85 & $18: 0-22: 4(n-6)$ \\
\hline & & 0.73 & 0.75 & $18: 0-20: 3(n-6)$ \\
\hline 13 & 21.87 & 9.03 & 9.22 & $16: 0-16: 0$ \\
\hline 14 & 23.52 & $\operatorname{Tr}$ & $\operatorname{Tr}$ & $18: 0-20: 3(n-9)$ \\
\hline 15 & 25.15 & 4.31 & 4.40 & $18: 1-20: 1$ \\
\hline 16 & 27.25 & 4.31 & 4.40 & $18: 0-18: 1$ \\
\hline 17 & 29.25 & 3.71 & 3.78 & $16: 0-18: 0$ \\
\hline 18 & 35.50 & $\operatorname{Tr}$ & $\operatorname{Tr}$ & 18:0-20:1 \\
\hline Total & & $98.00 \mathrm{nmol}$ & 100.01 & \\
\hline
\end{tabular}

Total rat brain DAG, after isolation by TLC, was benzoylated, purified, and subjected to HPLC as described in the text. The peak numbers correspond to the separation profile as presented in Fig. 2. Each peak from the HPLC column was collected, and its fatty acid composition was determined by GLC analysis as described in the text. When more than one species of DAG was present in the peak (more than two fatty acids), the assignment of species was done from the ratio of each fatty acid and the hydrophobicity of each species as described in the text. In the identified peaks the first number indicates the length of the carbon chain and the second the number of double bonds in the chain. In the assignment of the position of the fatty acid, it was arbitrarily assumed that the more unsaturated fatty acid was positioned at $\mathrm{C}-2$. $\mathrm{Tr}$, trace.

in our tests, 4-PP proved to be more effective than dimethylaminopyridine in terms of complete acylation of DAG in a shorter interval without any migration of the acyl groups. Distearoyl glycerol was used as the internal standard, because this moiety is virtually absent from tissue glycerides, and on HPLC it did not overlap with any other molecular species. The use of a fully saturated internal standard, however, raised the question of properly compensating for the loss of species containing unsaturated fatty acyl moieties during the purification of DAG. However, when tested, the total recovery of a species containing highly unsatu-

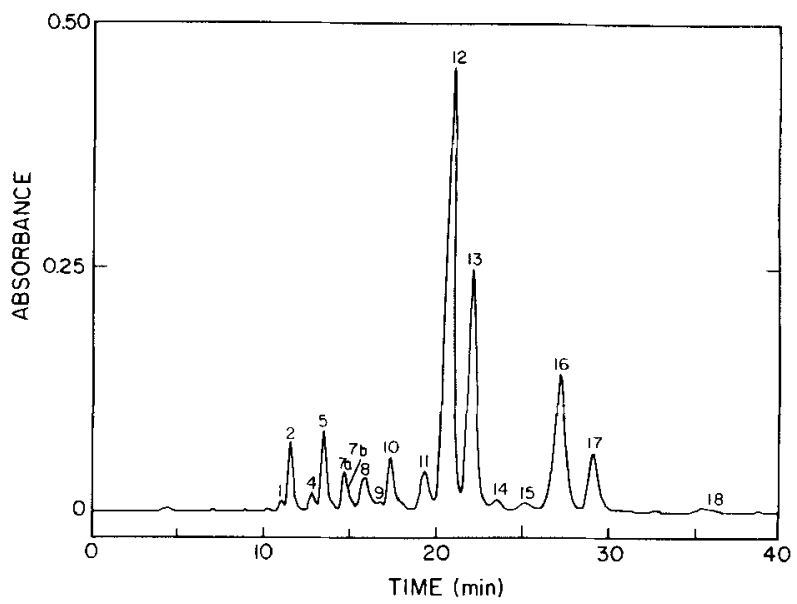

FIG. 3. Molecular species profile of rat brain PC. Rat brain PC was hydrolyzed with phospholipase $C$, and the resulting DAG was benzoylated, purified, and subjected to HPLC separation as described in the text. About $200 \mathrm{nmol}$ of benzoyl derivatives obtained from PC was injected into the HPLC column. The peak numbers correspond to the same numbers as in Fig. 2.

rated 20:4 (Table 1) was fairly close to the recovery of distearoyl glycerol ( 72 vs. $73 \%$ ), a result indicating that the comparative loss of DAG containing polyunsaturated fatty acids was $<2 \%$. The loss of unsaturated

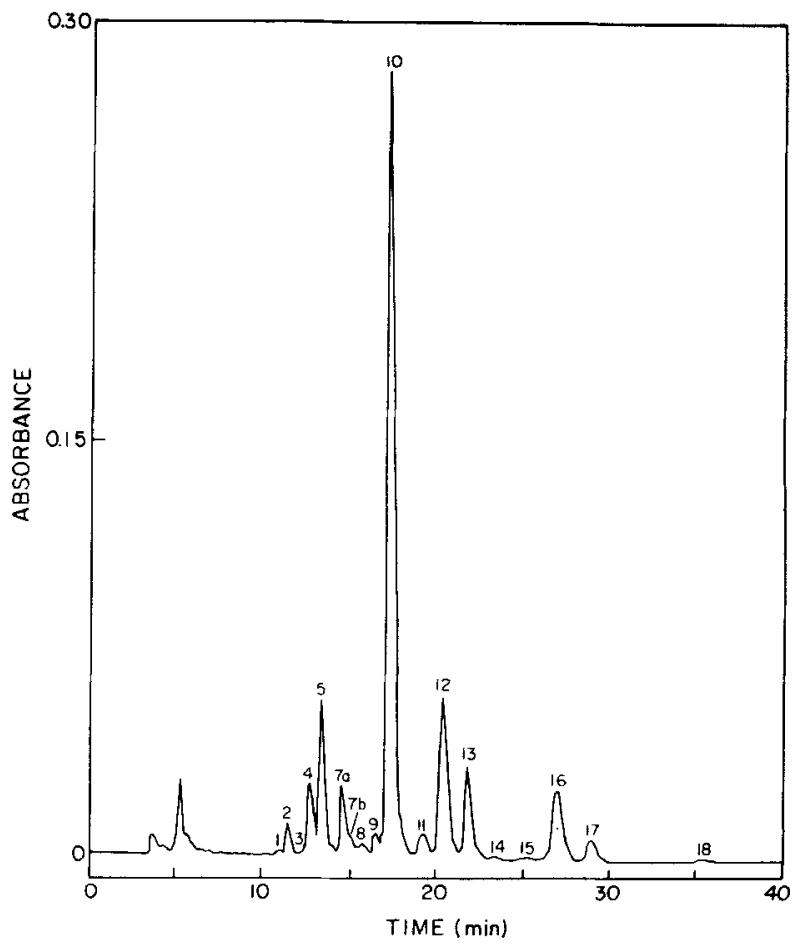

FIG. 4. HPLC separation profile of molecular species of PI in rat brain. Rat brain (1 min postmortem) PI was isolated and hydrolyzed to DAG, which was then benzoylated, and the molecular species were separated from each other by reverse-phase HPLC as described in the text. About $100 \mathrm{nmol}$ of benzoyl DAG obtained from PI was injected into the HPLC column. The numberings correspond to those of the HPLC profile shown in Fig. 2 and Table 2. 


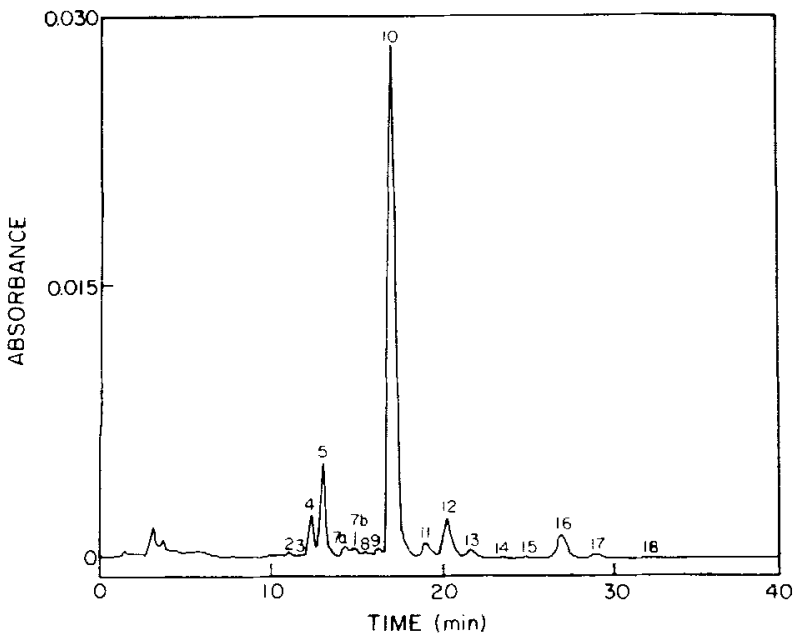

FIG. 5. HPLC separation profile of molecular species of $P P_{2}$ in rat brain. Rat brain $\mathrm{PIP}_{2}$ was isolated, dephosphorylated to $\mathrm{PI}$, and hydrolyzed to DAG, which was then benzoylated and used for HPLC separation as described in the text. Ten nanomoles of benzoyl DAG obtained from $\mathrm{PIP}_{2}$ was used for the separation. The numberings correspond to Fig. 2 and Table 2.

moieties was minimized by rapid processing and, when feasible, conducting the operations under a $\mathrm{N}_{2}$ atmosphere. The rapid processing also prevented any acyl migration (1,2-diacylglycerol to 1,3-diacylglycerol) before the benzoylation step. If the crude lipid extract or purified DAG was stored for long intervals, especially above $\mathrm{pH} 7$, acyl migration occurred, as seen by the presence of the 1,3-isomers as shoulders to the 1,2diacyl peaks in the HPLC separation profile.

The validity of the internal standard method of quantification has been verified against two other quantitative methods. Similar amounts of DAG were obtained when measurements were made after acetylation of the purified DAG with $\left[{ }^{14} \mathrm{Clacetic}\right.$ anhydride of known specific activity (D. DeMarais, C. Lee, and A. K. Hajra, unpublished data). Also, with the TLCpurified DAG, similar results were obtained when enzymatic phosphorylation by $\left[\gamma^{32} \mathrm{P}\right] \mathrm{ATP}$, as described by Preiss et al. (1986), was used in two experiments ( 93 and $96 \mathrm{nmol} / \mathrm{g}$ of brain as estimated by the above HPLC method vs. 93 and $91 \mathrm{nmol} / \mathrm{g}$ of brain when the enzymatic method was used). ${ }^{1}$

The molecular species composition of DAG (Fig. 1 and Table 2) reveals that $18: 0-20: 4$ (peak 10) is the

\footnotetext{
' We found that for the enzymatic estimation, DAG from total brain lipid extract should be first purified before phosphorylating with ATP using DAG kinase (Lipidex). When the measurement was done using total brain lipid extract, the amount of DAG was twoto threefold higher than that determined by the benzoylation or acetylation reactions. These high values were probably due to the presence of PA phosphatase in the commercial DAG kinase (crude Escherichia coli membranes), which hydrolyzes the large amounts of PA present in the brain lipid extracts to DAG. TLC purification of DAG removed the PA, and reproducible results were obtained using the enzymatic phosphorylation method.
}

TABLE 3. Distribution profile of the individual molecular species $P I, P I P, P I P_{2}, P C, P E$, and $P S$

\begin{tabular}{ccccccc}
\hline Peak & PI & PIP & PIP $_{2}$ & PC & PE & PS \\
\hline 1 & 0.18 & 0.09 & $\mathrm{Tr}$ & 0.46 & 1.01 & 0.14 \\
2 & 1.43 & 0.55 & 0.15 & 3.35 & 4.85 & 0.80 \\
3 & 0.09 & 0.10 & 0.54 & $\mathrm{Tr}$ & $\mathrm{Tr}$ & 0.34 \\
4 & 3.87 & 4.09 & 4.35 & 0.95 & 2.92 & 0.10 \\
5 & 7.84 & 9.38 & 9.54 & 4.42 & 2.30 & 0.61 \\
6 & $\mathrm{Tr}$ & $\mathrm{Tr}$ & $\mathrm{Tr}$ & $\mathrm{Tr}$ & 0.26 & 0.22 \\
$7 \mathrm{a}^{a}$ & & 1.52 & 1.09 & & & \\
& 4.08 & & & 2.49 & 17.62 & 42.40 \\
$7 \mathrm{~b}^{a}$ & & 1.20 & 0.96 & & & \\
8 & 0.59 & 0.82 & 0.38 & 3.06 & 1.47 & 0.78 \\
9 & 0.96 & 1.12 & 0.69 & 0.42 & 0.24 & 5.27 \\
10 & 49.52 & 63.72 & 66.13 & 3.84 & 22.49 & 3.76 \\
11 & 1.67 & 4.49 & 2.14 & 3.43 & 11.10 & 7.01 \\
12 & 12.67 & 5.94 & 6.54 & 36.18 & 15.80 & 9.07 \\
13 & 6.90 & 1.22 & 1.37 & 19.17 & 0.68 & $\mathrm{Tr}$ \\
14 & 0.32 & 0.12 & 0.11 & 0.94 & 0.52 & $\mathrm{Tr}$ \\
15 & 0.40 & 0.49 & 0.40 & 0.91 & 1.41 & 2.48 \\
16 & 7.04 & 4.15 & 4.60 & 14.13 & 14.85 & 23.67 \\
17 & 1.96 & 0.93 & 0.98 & 5.38 & 1.19 & $\operatorname{Tr}$ \\
18 & 0.50 & $\mathrm{Tr}$ & $\mathrm{Tr}$ & 0.71 & 1.30 & 2.22 \\
\hline
\end{tabular}

Data are mol\% of each molecular species. Peak numbers match with those in Figs. 2-4. Tr, trace.

${ }^{a}$ For PI, PC, PE, and PS, 7b appeared as a shoulder on peak 7a and was not resolved from the larger peak. Therefore, these values represent combined $7 \mathrm{a}$ and $7 \mathrm{~b}$.

main component, a finding indicating that inositides are the precursor for this species. However, peaks corresponding to other lipids (mainly PC), such as 12 (16:0-18:1) and $13(16: 0-16: 0)$, are also present. The molecular species composition agrees well with the fatty acid composition of rat or mouse brain DAG reported from different laboratories (Sun, 1970; Aveldano and Bazan, 1975; Ikeda et al., 1986). From the fatty acid

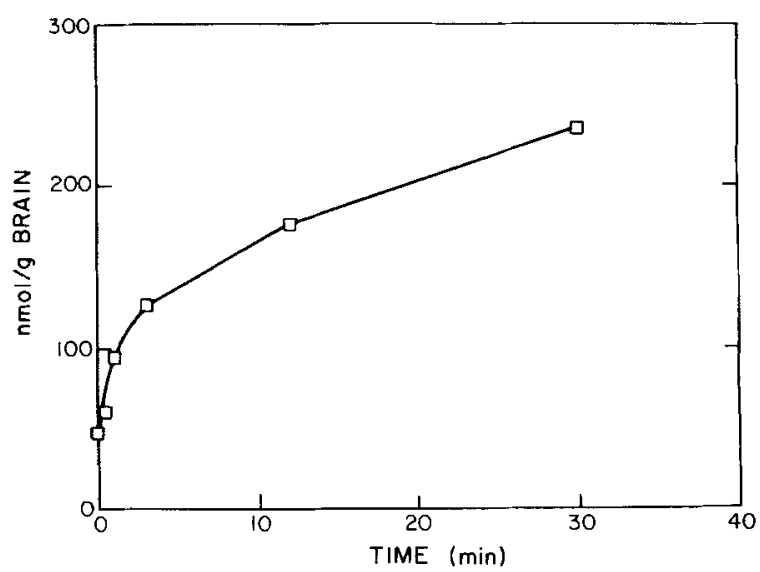

FIG. 6. Postmortem increase in amount of total DAG in rat brain. DAG was isolated, benzoylated, and then subjected to HPLC as described in the text. The total amount (average of two experiments, which were within $5 \%$ of each other) was calculated from the areas under the peaks and comparable area under the internal standard (18:0-18:0) peak. 


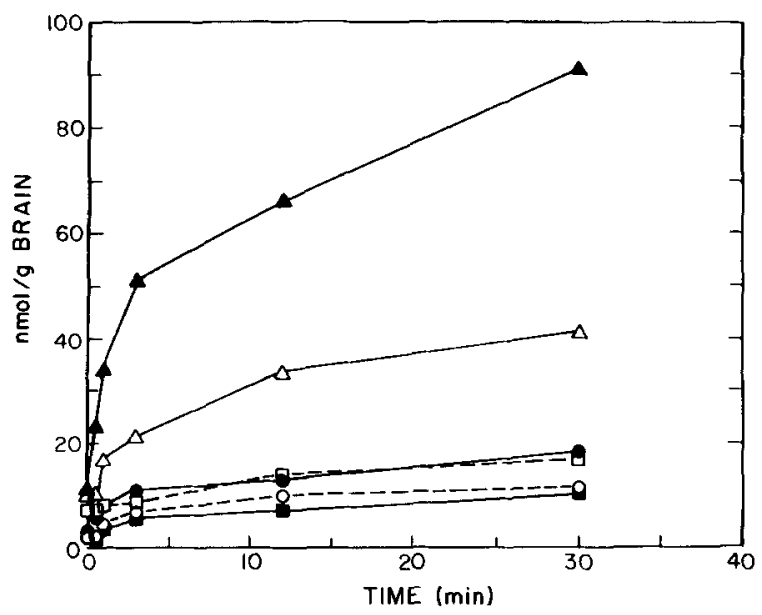

FIG. 7. Postmortem changes in the molecular species of rat brain DAG. Anesthetized rats were decapitated, and the brain DAG was extracted and analyzed at different intervals as described in the text. The changes of the amount of major DAG species in rat brain

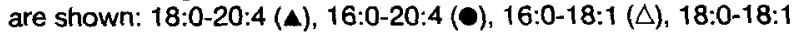
(O), 18:0-22:6 (a), and 16:0-16:0(口).

compositions, however, the actual molecular species of different lipids could not be accurately inferred. For example, it is generally assumed that all the stearic acid corresponds only to the arachidonate at $\mathrm{C}-2$, which leads to overestimation of the 18:0-20:4 species. As seen in Fig. 2, although 18:0-20:4 is the major one, there are other arachidonate-containing species, such as 16:0-20:4 (peak 5;8\%) and 18:1-20:4 (peak 4; 4\%). Similarly, there are several species containing stearic acid (peaks 10-18) but no arachidonic acid (see Table 2). Therefore, it is not possible to deduce such a complex molecular composition of DAG or other lipids from the total fatty acid composition of the lipids.

The HPLC method, which separated different molecular species of benzoyl DAG very well, was used to analyze the molecular species of most brain phosphoglycerides (diacyl species), which was not done before. As shown in Table 3, qualitatively the same molecular species are present in all phosphoglycerides and DAG. However, their quantitative compositions were quite different from one class of lipid to another. For example, in PS and PE (especially in PS), the species containing 22:6 (peak 7) was present in large amounts but was practically absent in PC. On the other hand, the dipalmitoyl moiety (peak 13) was present in large amounts in PC but not in PS or PE (Table 3). As expected, the 18:0-20:4 species was the predominant one in the inositides. However, although the compositions of PIP and PIP ${ }_{2}$ were very close to one another, indicating a precursor-product relationship, the composition of PI was somewhat different (Table 3). PI contained comparatively greater proportions of peaks 12 , 13 , and 16 , which consisted mainly of saturated and monounsaturated fatty acids (Table 2 ). This difference in composition of PI from PIP and $\mathrm{PIP}_{2}$ probably reflects the fact that the fatty acid composition of de novo biosynthetic PI is mainly saturated and monounsaturated, which is later tailored to the 18:0-20:4 and similar species (Holub and Kuksis, 1978). This latter PI pool (in the plasma membrane?) is probably the precursor of PIP and PIP ${ }_{2}$. Another finding of potential interest

TABLE 4. Postmortem changes in the total and individual amount of rat brain DAG

\begin{tabular}{|c|c|c|c|c|c|c|c|c|c|c|}
\hline \multirow[b]{2}{*}{ Peak } & \multicolumn{2}{|c|}{$0 \min ^{a}$} & \multicolumn{2}{|c|}{$0.5 \mathrm{~min}$} & \multicolumn{2}{|c|}{$1 \mathrm{~min}$} & \multicolumn{2}{|c|}{$12 \mathrm{~min}$} & \multicolumn{2}{|c|}{$30 \mathrm{~min}$} \\
\hline & $\mathrm{nmol} / \mathrm{g}$ & $\%$ & $\mathrm{nmol} / \mathrm{g}$ & $\%$ & $\mathrm{nmol} / \mathrm{g}$ & $\%$ & $\mathrm{nmol} / \mathrm{g}$ & $\%$ & $\mathrm{nmol} / \mathrm{g}$ & $\%$ \\
\hline 1 & 0.17 & 0.37 & 0.26 & 0.44 & 1.25 & 1.36 & 0.85 & 0.48 & 2.50 & 1.00 \\
\hline 2 & 1.64 & 3.63 & 1.81 & 3.10 & 2.93 & 3.18 & 5.22 & 2.93 & 12.31 & 4.93 \\
\hline 3 & 0.33 & 0.73 & Tr & $\operatorname{Tr}$ & $\operatorname{Tr}$ & $\operatorname{Tr}$ & 0.16 & 0.09 & 0.25 & 0.10 \\
\hline 4 & 1.19 & 2.64 & 1.49 & 2.54 & 3.18 & 3.45 & 3.42 & 1.92 & 6.74 & 2.70 \\
\hline 5 & 2.57 & 5.70 & 4.19 & 7.16 & 5.66 & 6.15 & 10.03 & 5.63 & 17.03 & 6.82 \\
\hline 6 & $\mathrm{Tr}$ & $\operatorname{Tr}$ & 0.23 & 0.39 & $\operatorname{Tr}$ & $\operatorname{Tr}$ & $\operatorname{Tr}$ & $\operatorname{Tr}$ & $\mathrm{Tr}$ & $\mathrm{Tr}$ \\
\hline 7 & 1.21 & 2.69 & 1.08 & 1.85 & 2.32 & 2.52 & 5.57 & 3.13 & 10.84 & 4.34 \\
\hline 8 & 2.26 & 5.00 & 1.57 & 2.68 & 3.00 & 3.21 & 4.02 & 2.26 & 6.67 & 2.67 \\
\hline 9 & 1.49 & 3.31 & 1.79 & 3.06 & 1.44 & 1.56 & 3.35 & 1.88 & 5.69 & 2.28 \\
\hline 10 & 9.82 & 21.78 & 19.11 & 32.67 & 33.07 & 35.91 & 61.99 & 34.81 & 92.90 & 37.21 \\
\hline 11 & 1.39 & 3.09 & 1.47 & 2.51 & 4.59 & 4.99 & 6.02 & 3.38 & 7.02 & 2.81 \\
\hline 12 & 9.58 & 21.24 & 10.34 & 17.68 & 16.20 & 17.59 & 38.52 & 21.63 & 45.76 & 18.33 \\
\hline 13 & 6.88 & 15.26 & 7.39 & 12.63 & 7.90 & 8.58 & 15.97 & 8.97 & 19.15 & 7.67 \\
\hline 14 & $\mathrm{Tr}$ & $\operatorname{Tr}$ & 0.50 & 0.86 & $\mathrm{Tr}$ & $\operatorname{Tr}$ & $\mathrm{Tr}$ & $\mathrm{Tr}$ & 0.22 & 0.09 \\
\hline 15 & 0.32 & 0.71 & 0.47 & 0.81 & 0.99 & 1.08 & 1.60 & 0.90 & 1.37 & 0.55 \\
\hline 16 & 2.05 & 4.55 & 2.18 & 3.72 & 4.50 & 4.89 & 10.63 & 5.97 & 10.96 & 4.39 \\
\hline 17 & 3.93 & 8.71 & 4.43 & 7.58 & 5.07 & 5.51 & 10.03 & 5.63 & 10.26 & 4.11 \\
\hline 18 & 0.24 & 0.54 & 0.12 & 0.20 & $\mathrm{Tr}$ & $\mathrm{Tr}$ & 0.16 & 0.09 & $\operatorname{Tr}$ & $\operatorname{Tr}$ \\
\hline Total & 45.11 & & 58.50 & & 92.08 & & 177.52 & & 249.67 & \\
\hline
\end{tabular}

At each interval, the first column is the concentration ( $\mathrm{nmol} / \mathrm{g}$ of brain), and the second column is the mol\% of the individual molecular species. Peak numbers correspond to those in Fig. 2. Tr, trace.

${ }^{a}$ The actual time may be $\sim 10-15 \mathrm{~s}$ postmortem. 


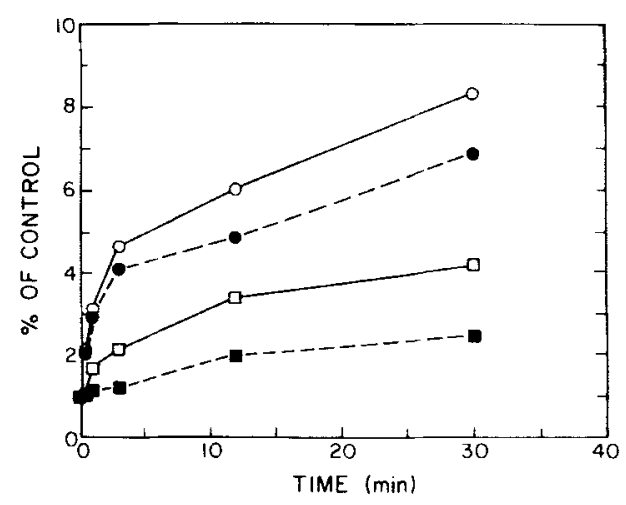

FIG. 8. Relative changes in amount of some individual DAG molecular species in rat brain. The relative amounts of some molecular species of DAG at each time point compared with the amount present at zero-time are plotted: 18:0-20:4 (O), 16:0-20:4 (๑), 16:

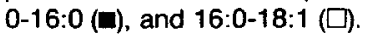

is that although brain PE and PS compositions are similar (Table 3), indicative of a precursor-product relationship, a much larger amount of 22:6 (peak 7a) and much lesser amount of 20:4 (peak 10) are present in PS than in PE. This may be due to compartmentation of different molecular species of PE and the formation of PS from PE in a particular compartment by the base exchange reaction.

The rapid postmortem increase in amount of DAG (Fig. 7) is similar to those reported by other authors (Banschbach and Geison, 1974; Ikeda et al., 1986). From the fatty acid analysis of the DAG formed, it is generally believed that this rapid increase in the concentration of DAG is entirely due to the breakdown of inositides. However, analysis of the molecular species indicated that not only the inositides but also PC was breaking down to DAG, albeit at a different rate. The species from inositides such as 16:0-20:4 and 18:0-20: 4 rapidly increased in content within 1-3 min after death, whereas those coming from PC, e.g., 16:0-18:1, also increased in content but at a slower rate. This was clearly seen when the relative rates of increase of different species were considered (Fig. 8). As seen in Fig. 8 , there is a rapid increase in content of 18:0-20:4 and 16:0-20:4 in the first 2 min followed by a relatively slower rate of increase for these two species, which are typical for inositol-containing lipids. There was a simultaneous rapid postmortem decrease in content of PIP and PIP 2 (Dawson and Eichberg, 1965; Ikeda et al., 1986; authors' unpublished data), a result indicating a phospholipase C-mediated breakdown of these lipids. A slower but steady and parallel rate of increase in content of 16:0-18:1 and 16:0-16:0 species, which are typical of brain PC (Table 3), is seen, which indicates that there is also a postmortem breakdown of brain $P C$ to DAG.

The present work was undertaken to establish whether or not the source and nature of DAG formed in a biological system could be determined by analyzing the molecular species of DAG in different biological systems so that the messenger and biosynthetic roles of DAG are delineated. The method presented here is fairly sensitive, so that $3-5 \mathrm{nmol}$ of total DAG (300 pmol of individual components) can be quantitatively measured in tissues or in incubation mixtures. The method is tested by studying the postmortem changes of molecular species of DAG in brain, and, as shown here, specific lipids are found to break down at different rates to form DAG. The results are, of course, not easily interpreted with respect to actual source and compartments where these transformations have taken place, because whole brain constitutes several different cell types and subcellular compartments. Using specific cell types and/or subcellular fractions might yield better results with respect to the precursor-product relationship of DAG and specific lipids and also the metabolic fate of the DAG formed. We have used this method to study the formation of DAG in SK-N-SH neuroblastoma cells after muscarinic stimulation and obtained results showing a rapid formation of DAG from inositides but not from PC in these cells (C. Lee, S. K. Fisher, B. W. Agranoff, and A. K. Hajra, manuscript in preparation). These and other studies are in progress in our laboratory to study the detailed mechanisms of formation and turnover of DAG, which acts both as a precursor of lipids and as an effector of signal transductions in most biological systems.

Acknowledgment: This work was supported by grant NS 008841 from the National Institutes of Health and grant $\mathrm{MH}$ 42652 from the National Institute of Mental Health.

\section{REFERENCES}

Aveldano M. I. and Bazan N. G. (1975) Rapid production of diacylglycerols enriched in arachidonate and stearate during early brain ischemia. J. Neurochem. 25, 919-920.

Banschbach M. W. and Geison R. L. (1974) Post-mortem increase in rat cerebrum hemisphere diacylglyceride pool. J. Neurochem. 23, 875-877.

Batley M., Packer N. H., and Redmond J. W. (1980) High performance liquid chromatography of diglyceride p-nitrobenzoates: an approach to molecular analysis of phospholipids. J. Chromatogr. 198, 520-525.

Bell R. M. and Coleman R. A. (1980) Enzymes of glycerolipid synthesis in eucaryotes. Annu. Rev. Biochem. 49, 459-487.

Blank M. L., Robinson M., Fitzgerald V., and Snyder F. (1984) Novel quantitative method for determination of molecular species of phospholipids and diglycerides. J. Chromatogr. 298, 473-482.

Dawson R. M. C. and Eichberg J. (1965) Diphosphoinositide and triphosphoinositide in animal tissues. Biochem. J. 96, 634-643.

Ehle H., Mueller E., and Horn A. (1985) Alkaline phosphatase of calf intestine hydrolyzes phospholipids. FEBS Lett. 183, 413416.

Exton J. H. (1990) Signaling through phosphatidylcholine breakdown. J. Biol. Chem. 265, 1-4.

Folch J., Lees M., and Sloane Stanley G. H. (1957) A simple method for the isolation and purification of total lipides from animal tissues. J. Biol. Chem. 226, 497-509.

Grove R. I., Fitzpatrick D., and Schimmel S. D. (1981) Effect of $\mathrm{Ca}^{++}$on triphosphoinositide extraction in fusing myoblasts. Lipids 16, 691-693. 
Hajra A. K., Fisher S. K., and Agranoff B. W. (1988) Isolation, separation and analysis of phosphoinositides from biological sources, in Lipids and Related Compounds (Boulton A. A., Baker G. B., and Horrocks L. A., eds), pp. 211-225. Humana Press, Clifton, New Jersey.

Hedegaard E. and Jensen B. (1981) Nano-scale densitometric quantitation of phospholipids. J. Chromatogr. 225, 450-454.

Holub B. J. and Kuksis A. (1978) Metabolism of molecular species of diacylglycerol-phospholipids. Adv. Lipid Res. 16, 1-125.

Ikeda M., Yoshida S., Busto R., Santiso M., and Ginsberg M. D. (1986) Polyphosphoinositides as a probable source of brain free fatty acids accumulated at the onset of ischemia. J. Neurochem. 47, 123-132.

Kennedy E. P. (1957) Biosynthesis of phospholipids. Fed. Proc. 16, $847-853$.

Kennerly D. A. (1987) Diacylglycerol metabolism in mast cells. $J$. Biol. Chem. 262, 16305-16313.

Keough K. M. W., Macdonald G., and Thompson W. (1972) A possible relation between phosphoinositides and the diglyceride pool in rat brain. Biochim. Biophys. Acta 270, 337-347.

Krueger J., Rabe H., Reichman G., and Rustow B. (1984) Separation and determination of diacylglycerols as their naphthylurethanes by high-performance liquid chromatography. $J$. Chromatogr. 307, 387-392.

Loeffelholz K. (1989) Receptor regulation of choline phospholipid hydrolysis. Biochem. Pharmacol. 38, 1543-1549.

Nakagawa Y. and Horrocks L. A. (1983) A separation of alkenylacyl, alkylacyl, and diacyl analogues and their molecular species by high performance liquid chromatography. J. Lipid Res. 24, 1268 1275.
Nishizuka Y. (1984) The role of protein kinase C in cell surface signal transduction and tumor promotion. Nature 308, 693-698.

Nishizuka Y. (1986) Turnover of inositol phospholipids and signal transduction. Science 225, 1365-1367.

Patton G. M., Fasulo J. M., and Robins S. J. (1982) Separation of phospholipids and individual molecular species of phospholipids by high-performance liquid chromatography. J. Lipid Res. 23, 190-196.

Pelech S. L. and Vance D. E. (1988) Signal transduction via phosphatidylcholine cycles. Trends Biol. Sci. 14, 28-30.

Preiss J., Loomis C. R., Bishop W. R., Stein R., Niedel J. E., and Bell R. M. (1986) Quantitative measurement of $s n$-1,2-diacylglycerols present in platelets, hepatocytes, and ras and sis-transformed normal rat kidney cells. J. Biol. Chem. 261, 8597-8600.

Prescott S. M. and Majerus P. W. (1983) Characterization of 1,2diacylglycerol hydrolysis in human platelets. J. Biol. Chem. 258, 764-769.

Sun G. Y. (1970) Composition of acyl groups in the neutral glycerides from mouse brain. $J$. Neurochem. 17, 445-446.

Sundler R., Alberts W. A., and Vagelos P. R. (1978) Enzymatic properties of phosphatidylinositol inositolphosphohydrolase from Bacillus cereus. J. Biol. Chem. 253, 4175-4179.

Van Rooijen L. A. A., Hajra A. K., and Agranoff B. W. (1985) Tetraenoic species are conserved in muscarinically enhanced inositide turnover. J. Neurochem. 44, 540-543.

Wright R. S. (1971) A reagent for the non-destructive location of steroids and some ether lipophilic materials on silica gel thinlayer chromatograms. J. Chromatogr. 59, 220-221. 